\title{
Synthesis and Evaluation of Important Biologically Active Heterocyclic Compounds: Schiff Bases, Oxadiazole and Pyrazolyl Derivatives
}

\author{
Mona A. Hosny ${ }^{a}$, Wafaa A. Mokbel ${ }^{a}$ and Emtithal A. El-Sawi ${ }^{a}$ \\ ${ }^{a}$ Department of Chemistry, Faculty of Women for Arts Science and Education, \\ Ain Shams University, Cairo, Egypt. \\ monaaminhosny@yahoo.com; wafaamokbel@ymail.com; elsawi_e@yahoo.com
}

\begin{abstract}
In this work, we prepared an excellent yield of (2-oxo-2H-pyrano[3,2-h] quinolin-4-yl) acetic acid; compound (1) and from the reaction of it with hydrazine hydrate $(100 \%)$ we obtain $2-(2-0 x o-2 H$-pyrano [3,2-h] quinolin-4-yl) aceto hydrazide (2) which is the starting material for the synthesis of several series of new compounds: such as schiff's bases (3a-e) and compound (4) in good yields, hydrazide derivatives like compound (5), derivatives of mercapto oxadiazole as compound (6) and derivatives of pyrazolyl as compound (7). All these compounds were found to possess high antimicrobial activity against $\mathrm{G}+$ and $\mathrm{G}$ - bacteria and against antifungal as described in Figure 2. Anticancer activity was screened only for compound (1). The IC50 value of it against breast cancer was found $=6.83 \mu \mathrm{M}$ comparable with that of the drug of doxorubicin which has IC50 = 5.6. As a result these newly compounds from (1) to (7) are considerable as potent compounds for various pharmacological activities.
\end{abstract}

\section{Indexing terms/Keywords}

Schiff bases; oxadiazole; pyrazolyl; antimicrobial; anticancer activity

\section{Council for Innovative Research}

Peer Review Research Publishing System

Journal: Journal of Advances in Chemistry

Vol. 9, No. 2

editorjaconline@gmail.com

www.cirworld.org/journals 


\section{INTRODUCTION}

The development of new coumarin derivatives is one of the fundamental goals in medicinal chemistry. Recently coumarin (2-oxo-2H-chromene) derivatives are considerable antimicrobial [1,2] compounds widely used as well as antifungal [3,4] and anticancer [5] effect. Moreover the new hydrazides, shiff base, oxidazole, pyrazolyl derivatives produced from the reaction of coumarine derivative and different agents which are the compounds of concern were found to exhibit appreciable antimicrobial [6-9] and antifungal [10-12] activities. Furthermore, a number of substituted pyrazolyl are important biologically active heterocyclic compounds due to that pyrazole containing pharmacoactive agents which play important role in medicinal chemistry such as anti-inflammatory, antiparacitic [13], antitumor, antiviral [14,15] and anticonvulsant [15-24] activities.

The present work directed to synthesize new hydrazides and heterocyclic compounds like pyrazolyl and oxadiazole derivatives which revealed that the development of new organic compounds as new drugs attracted much attention in the several future economics.

\section{Results and Discussion}

In the present work, we synthesized new hydrazide derivatives, heterocycles and schiff's bases from (2-oxo-2H-pyrano[3, 2-h] quinolin-4-yl) acetic acid as starting material which was originally prepared by 8-hydroxy qninoline with acetonedicarboxylic acid in the presence of concentrated sulfuric acid. Applying the hydrazinolysis of $(2-0 \times 0-2 \mathrm{H}-\mathrm{pyrano}$ [3, 2-h] quinolin-4-yl) acetic acid (1) with $100 \%$ hydrazine hydrate in absolute ethanol under reflux, $2-(2-0 \times 0-2 \mathrm{H}-\mathrm{pyrano}$ [3,2-h] quinolin-4-yl) acetohydrazide (2) was prepared in good yields. The acetohydrazide (2) was then condensed with different aromatic aldehydes in ethanol / acetic acid to give the corresponding schiffs bases, $\mathrm{N}$-[(1E)-(2,4-dihydroxy phenyl)methylene]-2-(2-oxo-2H-pyrano[3,2-h] quinolin-4-yl) acetohydrazide (3a-e) in good yields (see Figure 2). And then by condensation of (2) with ethylacetoacetate without solvent gave the schiff base compound (4) in good yield followed by refluxing of (2) in formic acid for 5 hours to produce the hydrazide derivative compound (5).

On the other hand, by heating the carbohydrazide of (2) with CS2 in the presence of ethanolic potassium hydroxide to produce compound (6) and then upon condensation of (2) with acetylacetone in ethanol containing a catalytic amount of acetic acid afforded the corresponding pyrazolyl derivative compound (7) in good yield (see Figure 2).

\section{The future prospective}

Moreover, it was found that compound (1) has an isomer in a good yield as in Figure 1. and it has an excellent microbiological activity as in table (2). So, we are working on it in the present time regarding its chemical reactions and its effectiveness in treating different series of diseases like inflammation and tumors.

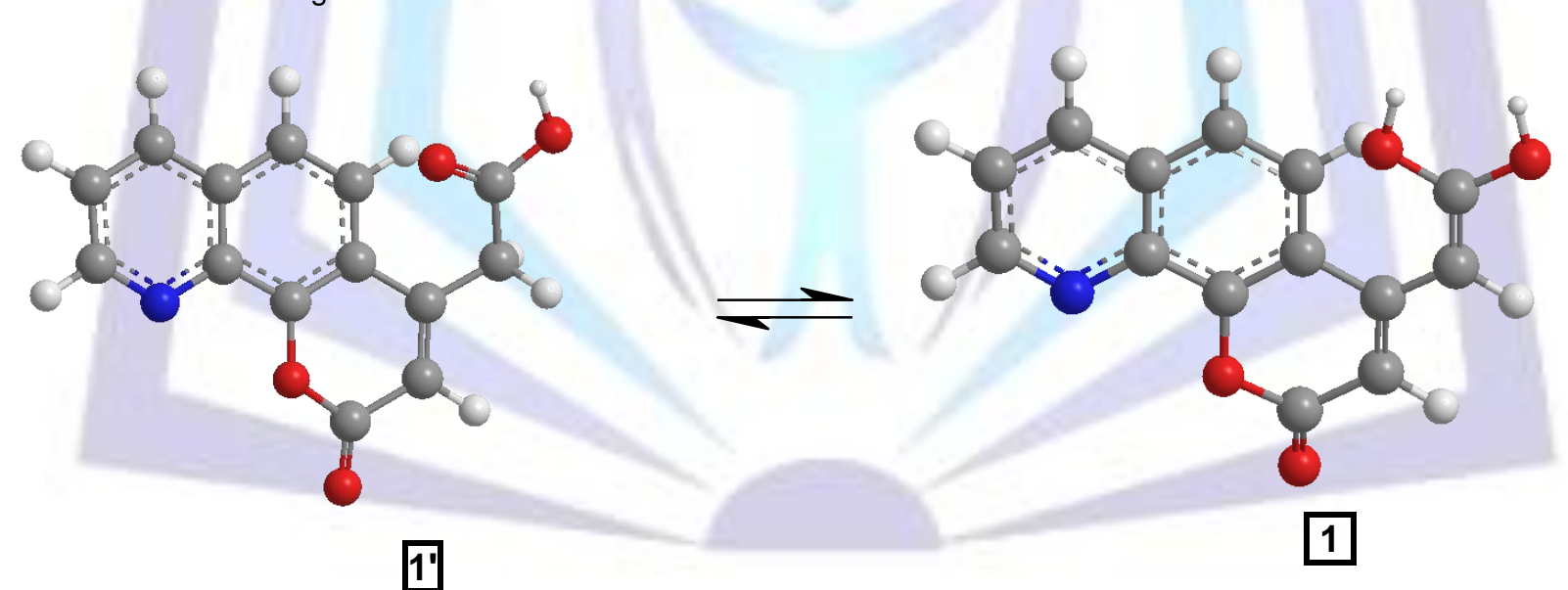

Figure 1 
Figure 2

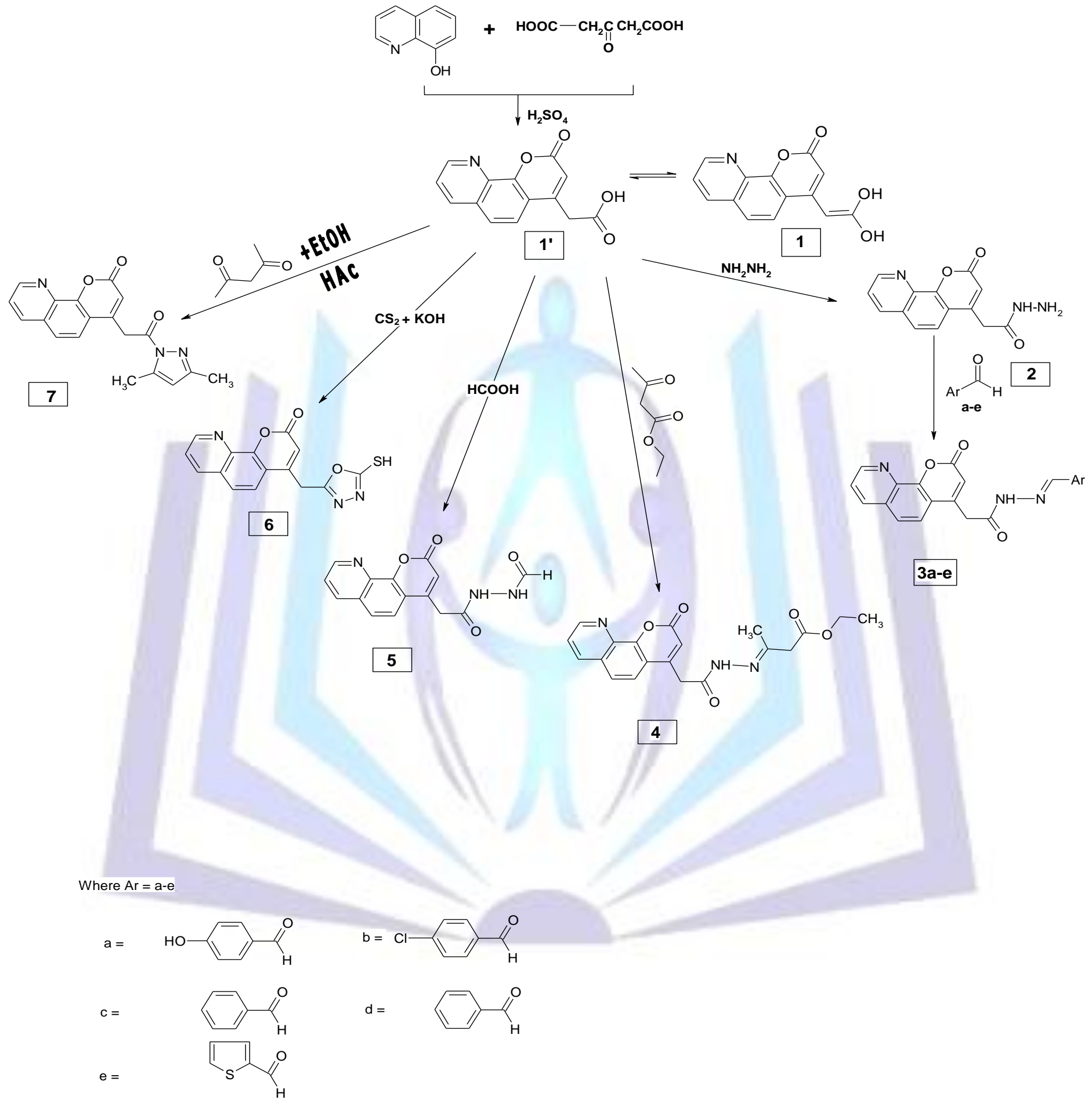




\section{Experimental section}

\subsection{General}

Melting points were taken on Gallen Kamp melting point apparatus and were uncorrected. Thin layer chromatography was performed with fluorescent silica gel plates HF254 (Merck), and plates were viewed under UV254 and 265 light. Infrared spectra $(\lambda$-cm-1) were recorded on Bruiker Vector Germany and on Mattson FT-IR 1000, using KBr disks, mass spectra were measured on GCO Finnigan MAT and 1H-NMR spectra were recorded Gemini $-300 \mathrm{MHZ}$ for $1 \mathrm{H}$ and $100 \mathrm{MHZ}$ for 13C respectively, in DMSO-d6 and TMS as internal standard and the antibacterial activity was determined in microanalytical center in main defense chemical laboratory of the Egyptian Accreditation council. Ethylaceto acetate, 1,3acetone dicarboxylic acid and different reactants were obtained from Fluka or Aldrich.

\section{2. (2-oxo-2H-pyrano [3,2-h] quinolin-4-yl) acetic acid (1)}

The phenol of 8-hydroxy qunioline with $(1 \mathrm{~g}, 10 \mathrm{mmol}$ was dissolved in $70 \%$ sulfuric acid (10 ml) at $0 \circ \mathrm{C}, 1,3-\mathrm{acetone}$ dicarboxylic acid $(19,10 \mathrm{mmol})$ was added in few portions. The mixture was allowed to warm up at room temperature and stirred further for 4 hours. The resulting solution was poured onto crushed ice then left in refrigerator for about two days. The formed yellow needles were collected by filtration and dried well under reduced pressure to afford (1) as yellow needles in 80\% yield; mp: 95oC, IR (KBr) (cm-1): $3505(\mathrm{OH}$, carboxylic acid), $3402(\mathrm{OH}$, enol form), 3077 (CH, aromatic), 2936 (CH2, methylene), 1723 (C=O, lactone), 1596-1553 ( $\mathrm{C}=\mathrm{N}) ; \mathrm{MS}(\mathrm{m} / \mathrm{z} \%), 256(\mathrm{M}+1,11.36 \%), 69(100 \%) ; 1 \mathrm{H}-\mathrm{NMR}$ (DMSO-d6) $\delta: 2.08(2 \mathrm{H}, \mathrm{CH} 2), 6.24(1 \mathrm{H}, \mathrm{CO}-\mathrm{CH}=), 7.46,8.05(5 \mathrm{H}, \mathrm{CH} \mathrm{Ar}), 9.10(\mathrm{H}, \mathrm{OH}) ; 13 \mathrm{C}-\mathrm{NMR}(100 \mathrm{MHZ}, \mathrm{DMSO}) ; \delta$ : $38.6,115.8,118.5,122.2,122.4,129.0,129.7,130.3,130.4,144.3,144.5,146.0,146.1,148.4$.

\subsection{2-(2-oxo-2H-pyrano [3,2-h] quinolin-4-yl) acetohydrazide (2)}

Compound $1(1 \mathrm{~g}, 1 \mathrm{mmol})$ was dissolved in a solution containing ethanol $(20 \mathrm{ml})$ and $10 \%$ hydrazine hydrate $(1 \mathrm{ml})$ and the mixture was refluxed for 4 hours. The product was separated, collected by suction filtration, washed with ethyl alcohol and recrystallized from ethylalcohol to give compound 2 as being ppt in $86 \%$ yield; mp: 65 oC, IR $(\mathrm{KBr})(\mathrm{cm}-1): 3450$ (NH2), hydrazide, 3049 (CH-aromatic), 1733 (C=O, lactone) 1624-1573 (-CO-NH-NH2 group); MS (m/z \%) = $269(\mathrm{M}+$, 2.13\%), 117 (100\%), 1H-NMR (DMSO-d6), $\delta: 2.08(2 \mathrm{H}, \mathrm{NH} 2), 3.51(2 \mathrm{H}, \mathrm{CH} 2), 7.07(1 \mathrm{H}, \mathrm{CO}-\mathrm{CH}=), 7.19-8.85(5 \mathrm{H}, \mathrm{Ar})$, $8.33(\mathrm{CH}$, quinoline), $9.77(1 \mathrm{H}, \mathrm{NH}) ; 13 \mathrm{C}-\mathrm{NMR}(100 \mathrm{MHZ}, \mathrm{DMSO}) ; \delta: 38.9,111.3,117.6,117.7,121.7,127.4,128.7$, $135.8,136.1,138.3,138.4,147.9,148.1,153.2$.

\subsection{General procedure for the preparation of N-[(1E)- (2,4-dihydroxy phenyl) methylene]-2-(2- oxo-2H-pyrano [3,2-h] quinolin-4-yl) acetohydrazide 3a-e}

A mixture of compound $2(1 \mathrm{~g}, 1 \mathrm{mmol})$ and the suitable aromatic aldehyde a-e $(1 \mathrm{mmol})$ was refluxed in absolute ethanol for 3 hours in presence of catalytic amount of glacial acetic acid. The excess of solvent was then removed under reduced pressure, the precipitate formed after cooling was collected by filtration and recrystalized from ethyl alcohol to give the different hydrazides $3 a-e$.

4.4.1. Compound (3a): Pale brown ppt., yield: $76 \%$; mp: $170 \circ \mathrm{C}$, IR (KBr) (cm-1): $3727(\mathrm{OH}$, free), $3465(\mathrm{NH}), 3213$ ( $\mathrm{CH}$, aromatic), 2931 (CH2, methylene), 1730 (C=O, lactone), 1616 (CO-NH-); MS (m/z\%): 389 (M+, 23.42\%), 117 (100\%); 1 H-NMR (DMSO-d6); $\delta$ : 3.52 (CH2, methylene), $6.32(1 \mathrm{H}, \mathrm{CH}$ lactone), $6.39(2 \mathrm{H}, \mathrm{CH}$ aromatic), $7.07-8.85(5 \mathrm{H}$, aromatic protons), $8.30(1 \mathrm{H},-\mathrm{N}=\mathrm{CH}-), 8.76(1 \mathrm{H}$, quinoline), $9.80(1 \mathrm{H}, \mathrm{NH}), 10.16(1 \mathrm{H}, \mathrm{OH}), 11.37(1 \mathrm{H}, \mathrm{OH}) ; 13 \mathrm{C}-\mathrm{NMR}$ (100MHZ, DMSO); $\delta: 52.1,102.4,108.1,110.2,110.2,121.5,121.5,128.3,129.4,132.8,134.3,136.1,152.6,152.6$, 158.4, 160.6, 161.6, 161.9, 172.4, 207.3.

4.4.2. Compound (3b): Pale beige ppt., yield: $88 \%$; mp: $2180 \mathrm{C}, \mathrm{IR}(\mathrm{KBr})(\mathrm{cm}-1): 3450(\mathrm{NH}), 3120(\mathrm{CH}$, aromatic), 2933 (CH2, methylene), 1686 (C=O, lactone), 1626 (CO-NH-); MS (m/z\%): 392 (M+, 2.54\%), 165 (100\%); 1H-NMR (DMSO-d6) $\delta$ : $3.57(\mathrm{CH} 2$, methylene), $5.23(1 \mathrm{H}, \mathrm{CH}$ lactone $), 7.54-7.95(8 \mathrm{H}$, aromatic protons $), 7.95(1 \mathrm{H},-\mathrm{N}=\mathrm{CH}-), 7.96(1 \mathrm{H}$, quinoline) $8.7(1 \mathrm{H}, \mathrm{NH})$; 13C-NMR (100MHZ, DMSO); $\delta: 70.5,106.9,120.8,120.8,120.8,123.1,128.6,128.6,129.5$, 129.9, 129.9, 131.0, 131.0, 137.7, 137.7, 141.8, 150.3, 150.3, 154.1, 160.4, 166.3.

4.4.3. Compound (3c): Beige ppt., yield: 95\%; mp: 220oC, IR (KBr) (cm-1): $3417(\mathrm{NH}), 3039(\mathrm{CH}$, aromatic), 2922 (CH2, methylene) $1698(\mathrm{C}=\mathrm{O}$, lactone), $1620(\mathrm{C}=\mathrm{O}-\mathrm{NH}-)$; MS (M/z \%): $373(\mathrm{M}+, 2.41 \%), 240(100 \%) ; 1 \mathrm{H}-\mathrm{NMR}(\mathrm{DMSO}$, d6) $\delta: 3.52(\mathrm{CH} 2$, methylene), $6.95(1 \mathrm{H}, \mathrm{CH}$ lactone $), 6.97-8.60(8 \mathrm{H}$, aromatic protons $), 8.57(1 \mathrm{H},-\mathrm{N}=\mathrm{CH}-), 8.91(1 \mathrm{H}$, quninoline), $8.99(1 \mathrm{H}, \mathrm{NH}), 10.2(1 \mathrm{H}, \mathrm{OH})$; 13C-NMR (100-MHZ-DMSO) $\delta: 46.3, .116 .4,118.1,119.5,119.5,119.5,119.5$, $124.2,124.2,129.6,130.8,133.1,133.1,142.8,151.4,151.4,158.5,158.5,162.7,170.2$.

4.4.4. Compound (3d): Pale beige ppt., yield: $87 \%$; mp: $80 \circ \mathrm{C}, \mathrm{IR}(\mathrm{KBr})(\mathrm{cm}-1) 3400(\mathrm{NH}), 3050(\mathrm{CH}$, aromatic), 2938 $(\mathrm{CH} 2$, methylene), $1694(\mathrm{C}=\mathrm{O}$, lactone), $1616(\mathrm{C}=\mathrm{O}-\mathrm{NH}-)$; MS (m/z\%); $357(\mathrm{M}+, 0.70 \%), 145(100 \%) ; 1 \mathrm{H}-\mathrm{NMR}(\mathrm{DMSO}$, d6) $\delta: 3.60(2 \mathrm{H}$, methylene) $6.90(1 \mathrm{H}, \mathrm{CH}$ lactone $), 7.23-7.89(9 \mathrm{H}$, aromatic protons), $8.57(1 \mathrm{H},-\mathrm{N}=\mathrm{CH}-), 8.68(1 \mathrm{H}$, quinoline), $8.92(1 \mathrm{H}, \mathrm{NH})$; 13C-NMR (100-MHZ-DMSO) $\delta: 71.5,109.5,121.8,121.8 .123 .5,126.9,126.9,128.2,128.8$, $128.8,131.2,131.2,133.7,136.2,142.4,150.4,150.1,154.4,161.2,165.3$. 
4.4.5. Compound (3e): Beige ppt., yield: $89 \%$; mp: 140oC, IR (KBr) (cm-1): $3409(\mathrm{NH}), 3089(\mathrm{CH}$, aromatic), 2921 ( $\mathrm{CH}$, methylene), 1671 ( $\mathrm{C}=\mathrm{O}$, lactone), 1603 (C=O-NH-); MS (m/z\%) $363(\mathrm{M}+, 1.71 \%), 220(100 \%) ; 1 \mathrm{H}-\mathrm{NMR}(\mathrm{DMSO}$, d6) $\delta$ : $2.79(2 \mathrm{H}$, methylene), $6.90(1 \mathrm{H}, \mathrm{CH}$ lactone) $7.16-7.24(3 \mathrm{H}, \mathrm{CH}$ thiophene $), 7.51(1 \mathrm{H},-\mathrm{N}=\mathrm{CH}-), 7.62-8.83(4 \mathrm{H}$, aromatic), $8.29(1 \mathrm{H}$, quinoline), $8.92(1 \mathrm{H}, \mathrm{NH}), 13 \mathrm{C}-\mathrm{NMR}(100 \mathrm{MHZ}-\mathrm{DMSO}) \delta: 71.3,112.8,121.5,126.7,128.2,128.2$, $130.8,131.7,133.6,133.8,134.7,136.0,138.3,147.5,150.1,153.8,155.6,161.6,172.0$.

\section{Schiff base compound (4)}

A mixture of compound $2(1 \mathrm{~g}, 1 \mathrm{mmol})$ and ethyl acetoacetate $(1 \mathrm{~g}, 1 \mathrm{mmole})$ was condensed without solvent at $150 \mathrm{oC}$ for $10 \mathrm{~min}$. The reaction mixture was cooled and refluxed in ethyl alcohol about $(25 \mathrm{ml})$ for 2 hours. The precipitate formed after cooling was collected by filtration and recrystalized from ethyl alcohol afforded compound 4 as brownish red ppt, in 90\% yield; mp: 108oC, IR (KBr) (cm-1): $3438(\mathrm{NH}), 3203-3027(\mathrm{CH}$, aromatic), $2984(\mathrm{CH} 2$, methylene), $2827(\mathrm{CH}$, methyl group), 1699 (C=O, lactone), 1605(C=O-NH-); MS (m/z \%) $381(\mathrm{M}+, 7.30 \%), 130(100 \%) ; 1 \mathrm{H}-\mathrm{NMR}$ (DMSO-d6); $\delta: 1.08$ $(3 \mathrm{H}, \mathrm{CH} 3), 1.13(3 \mathrm{H}, \mathrm{CH} 3), 2.17(2 \mathrm{H}, \mathrm{CH} 2), 2.36(2 \mathrm{H}, \mathrm{CH} 2), 3.77(2 \mathrm{H}, \mathrm{CH} 2), 5.44(1 \mathrm{H}, \mathrm{CH}$ lactone $), 5.80(1 \mathrm{H}, \mathrm{NH}), 6.91-$ $8.80(4 \mathrm{H}$, aromatic), 8.92 (1H, CH quinoline); 13C-NMR (100 MHZ, DMSO); $\delta: 16.0,16.0,39.1,39.7,57.6,111.1,117.6$, $121.7,121.8,127.5,128.7,135.9,138.3,148.0,148.0,153.1,153.1,156.8,167.0,171.0$.

\section{Hydrazide derivatives compound (5)}

A solution of compound $2(1 \mathrm{~g}, 1 \mathrm{mmol})$ in formic acid was refluxed for 5 hours. The solvent was evaporated and the precipitate formed was collected by filtration and recrystalized from ethylalcohol to give 5 as beige ppt in $76 \%$ yield, mp: 250oC, IR (KBr) (cm-1): $3728(\mathrm{OH}$, free), $3121(\mathrm{CH}$, aromatic), 1621 (CO-NH, amide group), $1521(\mathrm{C}=\mathrm{N}) ; \mathrm{MS}(\mathrm{m} / \mathrm{z} \%)$

296, $(\mathrm{M}+, 8.95 \%), 397(\mathrm{M}+$

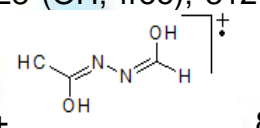

$=\mathrm{CH}-\stackrel{\mathrm{C}}{=}=\mathrm{N}), 5.12(1 \mathrm{H}$, lactone), $6.87(1 \mathrm{H}, \mathrm{OH}), 7.12-7.79(4 \mathrm{H}$, aromatic), $8.79(1 \mathrm{H}, \mathrm{CH}$ quinoline); 13C-NMR (100 MHZ, DMSO): $\delta: 71.08,105.1,106.2,115.1,121.4,121.4,124.4,126.5,137.1,138.6,149.7,150.1,156.4,158.6,205.4$.

\section{Mercapto-oxadiazole compound (6)}

To a mixture of acetohydrazide $2(1 \mathrm{~g}, 1 \mathrm{mmol})$ in ethyl alcohol $(50 \mathrm{ml})$ and a solution of potassium hydroxide $(0.5 \mathrm{~g}, 1$ $\mathrm{mmol})$ in ethyl alcohol $(5 \mathrm{ml})$ was added followed by carbon disulfide $(10 \mathrm{ml})$. The reaction mixture was heated under reflux for 6 hours, then it was concentrated, acidified with diluted $\mathrm{HCl}$ and the resulting solid was collected, washed with water and recrystalized from a mixture of DMFA-H2O to give compound 6 as deep brown ppt. in $94 \%$ yield; mp: $150 \circ \mathrm{C}$, IR $(\mathrm{KBr})(\mathrm{cm}-1)$ : $3432(\mathrm{C}=\mathrm{N}-\mathrm{N}), 3246-3011(\mathrm{CH}$, aromatic), $2849(\mathrm{CH} 2$, methylene), $2718(\mathrm{SH}), 1555(\mathrm{C}=\mathrm{N}), 1271(\mathrm{C}-\mathrm{S})$; MS (m/z\%): $311(\mathrm{M}+, 7.00 \%), 80(100 \%), 1 \mathrm{H}-\mathrm{NMR}$ (DMSO-d6); $\delta: 3.14(1 \mathrm{H}, \mathrm{SH}), 3.95(2 \mathrm{H}, \mathrm{CH} 2), 5.89(1 \mathrm{H}$, ethylene), 7.06-8.76 (4H, aromatic protons), $8.98(1 \mathrm{H}, \mathrm{CH}$ quinoline); 13C-NMR (100 MHZ-DMSO); $\delta: 38.6,70.2,111.4,118.0$, $118.0 ; 121.9,128.2,128.2,134.2,134.2,138.5,138.5,146.5,165.3,207.8$.

\section{Derivatives of pyrazolyl compound (7)}

A mixture of compound $2(1 \mathrm{~g}, 1 \mathrm{mmol})$, acetyl acetone $(1 \mathrm{~g}, 1 \mathrm{mmole})$ and acetic acid $(1.0 \mathrm{ml})$ was refluxed in ethyl alcohol $(10 \mathrm{ml})$ for 5 hours. The precipitate which formed after cooling was collected by filtration and recrystalized from ethyl alcohol to give compound 7 as deep brown ppt. in $87 \%$ yield; mp: $120 \circ \mathrm{C}$, IR $(\mathrm{KBr})(\mathrm{cm}-1): 3423(\mathrm{C}=\mathrm{N}-\mathrm{N}), 3196-3046(\mathrm{CH}$, aromatic), $2974(\mathrm{CH} 2$, methylene), $1696(\mathrm{C}=\mathrm{O}$, lactone), $1576(\mathrm{C}=\mathrm{N}), 1407,1377(\mathrm{CH}$, two methyl groups); MS (m/z \%): $321(\mathrm{M}+, 2.49 \%), 304$ (100\%); 1H-NMR (DMSO-d6); $\delta: 2.85(2 \mathrm{H}, 2 \mathrm{CH} 3), 3.26(2 \mathrm{H}$, methylene), 7.07 (1H, pyrazole), 7.08 $(1 \mathrm{H}, \mathrm{CH}$ lactone), 7.37-8.85 (4H, aromatic protons), 9.69 (1H, CH quinoline); 13C-NMR (100 MHZ-DMSO); $\delta: 28.1,28.2$, 42.3, 42.3, 43.9, 115.5, 120.6, 120.6, 120.6, 126.9, 126.9, 134.3, 135.1, 141.8, 150.3, 150.3, 156.1, $160.9,168.4$.

\section{Cytotoxicity}

The anticancer activity of the new synthesized compound (1) was tested against MCF-7 cancer cell lines using sulphorhodamine - B (SRB) assay following the method reported by Vichai and Kirtikara [25].

\subsection{Procedure}

1. Cells were seeded in 96 -well microtiter plates at initial concentration of $3 \times 103$ cell/well in a $150 \mu$ fresh medium and left for 24 hours to attach to the plates.

2. Different concentrations $0,5,12.5,25,50 \mu \mathrm{g} / \mathrm{ml}$ of drug were added.

3. For each drug concentration, 3 wells were used. The plates were incubated for 48 hours.

4. The cells were fixed with $50 \mu$ cold trichloroacetic acid $10 \%$ final concentration for 1 hour at $40 \mathrm{C}$.

5. The plates were washed with distilled water using (automatic washer Tecan, Germany) and stained with $50 \mu \mathrm{l} 0.4 \%$ SRB dissolved in $1 \%$ acetic acid for 30 minutes at room temperature.

6. The plates were washed with $1 \%$ acetic acid and air dried. 
7. The dye was solubilized with $100 \mu \mathrm{l} /$ well of $10 \mathrm{M}$ tris base $(\mathrm{pH} 10.5)$ and optical density (O.D) of each well was measured spectrophotometrically at $570 \mathrm{~nm}$ with an ELISA microplate reader (Sunrise Tecan reader, Germany). The mean background absorbance was automatically subtracted and mean values of each drug concentration was calculated. The experiment was repeated 3 times.

\subsection{Calculation}

The percentage of cell survival was calculated as follows:

Surviving fraction = O.D (treated cells)/ O.D. (control cells).

The $\mathrm{IC}_{50}$ values (the concentrations of resveratrol required to produce $50 \%$ inhibition of cell growth) were also calculated.

Compound (1) showed good evaluation against MCF-7 cancer cell lines, the $\mathrm{IC}_{50}$ value of it was found at $6.83 \mu \mathrm{g} / \mathrm{ml}$ comparable to the $\mathrm{IC}_{50}$ value of Doxoroubicien $5.6 \mu \mathrm{g} / \mathrm{ml}$ as in table (1), this result indicate that compound (1) and its derivatives may be useful leads for anticancer drug development in the future.

Table 3. Drug cytotoxicity of compound (1) and Doxorubicin

\begin{tabular}{c|c|c}
\hline Conc: $\mathbf{u g} / \mathbf{m l}$ & MCF7- 1 & MCF7-DOX \\
\hline 0.0 & 1.000000 & 1.0000 \\
5.0 & 0.515284 & 0.513889 \\
12.5 & 0.441485 & 0.380952 \\
25.0 & 0.318777 & 0.384921 \\
50.0 & 0.449782 & 0.326984 \\
\hline
\end{tabular}

Figure 3

MCF7

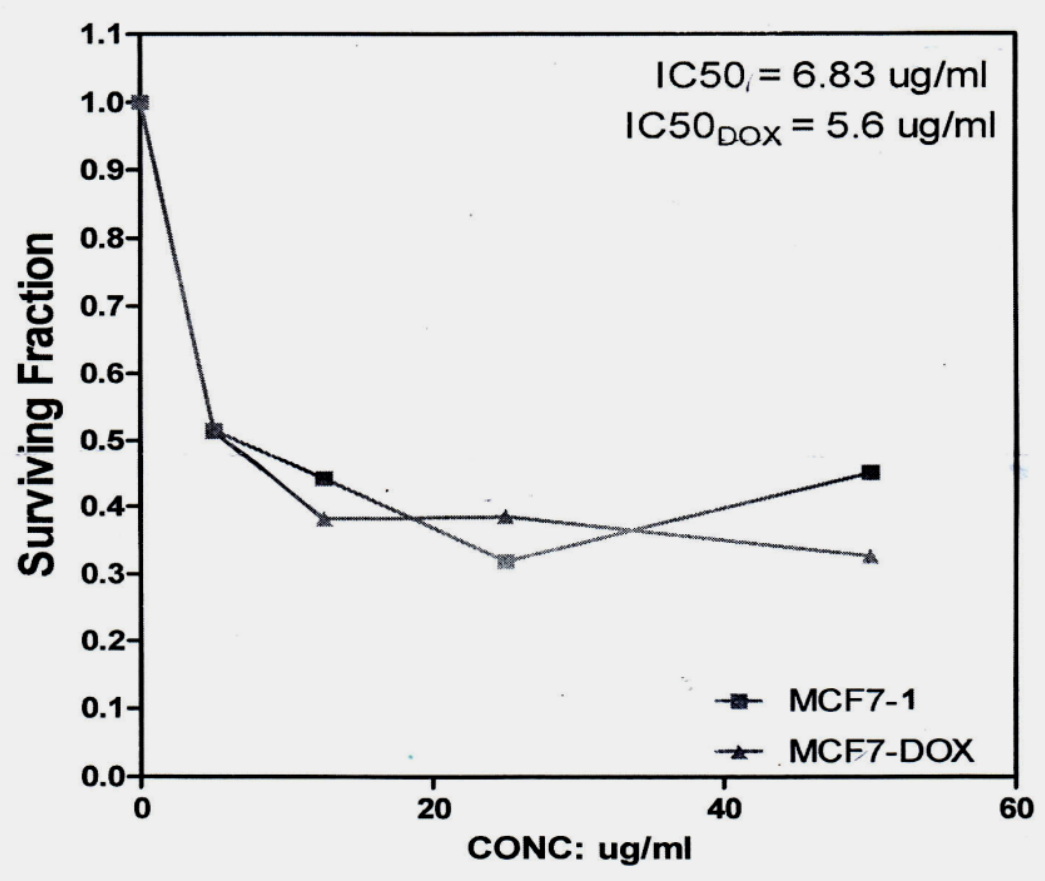




\section{Antimicrobial activity}

The newly prepared compounds especially compound 1 exhibited strong inhibition toward gram-positive bacteria (Staphylococcus aureus), gram - negative bacteria (Escherichia coli, Enterococcus fecalis) and antifungus (Aspergillus niger \& Candida albicans \& Yeast) as in table (2).

\subsection{Microbiological screening}

Antimicrobial activities of different extracts were evaluated by the agar well diffusion method [26] modified by Olurinola [27].

\subsection{Media preparation and its sterilization}

For agar well diffusion method [26] later modified by Olurinola [27] antimicrobial susceptibility was tested on solid (Agaragar) media in Petri plates. For bacterial assay nutrient agar (NA) (40 gm/L) and for fungus PDA (39 gm/L) was used for developing surface colony growth. The minimum inhibitory concentration (MIC) the minimum bactericidal concentration (MBC) and minimum fungicidal concentration (MFC) values were determined by serial micro dilution assay. The suspension culture, for bacterial cells growth was done by preparing $2 \%$ Lauria Broth $(\mathrm{w} / \mathrm{v})$, and for fungus cells growth, $2.4 \%(w / v)$ PDB (Potato dextrose broth) was taken for evaluation. All the media prepared was then sterilized by autoclaving the media at $(1210 \mathrm{C})$ for $20 \mathrm{~min}$.

\subsection{Agar well diffusion method}

Agar well diffusion method was followed to determine the antimicrobial activity. Nutrient Agar (NA) and Potato Dextrose Agar (PDA) plates were swabbed (sterile cotton swabs) with 8 hour old - broth culture of respective bacteria and fungi. Wells (10 mm diameter and about $2 \mathrm{~cm}$ a part) were made in each of these plates using sterile cork borer. Stock solution of each plant extract was prepared at a concentration of $1 \mathrm{mg} / \mathrm{ml}$ in different extracts. About $100 \mu \mathrm{l}$ of different concentrations of extracts were added by sterile syringe into the wells and allowed to diffuse at room temperature for 2 hrs. Control experiments comprising inoculums without extract were set up. The plates were incubated at $370 \mathrm{C}$ for $18-24 \mathrm{~h}$ for bacterial pathogens and $280 \mathrm{C}$ for 48 hours fungal pathogens. The diameter of the inhibition zone (mm) was measured and the activity index was also calculated. Triplicates were maintained and the experiment was repeated thrice, for each replicates the readings were taken in three different fixed directions and the average values were recorded.

The antimicrobial potential of both the experimental extracts was evaluated according to their zone of inhibition against various pathogens and the results (zone of inhibition) were compared with the activity of the standards, viz., Ampicillin (1.0 $\mathrm{mg} / \mathrm{disc})$, Flucanazole (1.0 mg/disc).

Table 4. Antimicrobial activity of the products towards some types of bacteria and fungus.

\begin{tabular}{|c|c|c|c|c|}
\hline \multirow{2}{*}{ Sample } & \multicolumn{4}{|c|}{ Inhibition zone in $\mathrm{mm}$ (conc. $\mu \mathrm{g} / \mathrm{ml})$} \\
\hline & Escherichia coli (G') & $\begin{array}{l}\text { Staphylococcus aureas } \\
\left(\mathrm{G}^{+}\right)\end{array}$ & Aspergillus nigar & Candida albicans \\
\hline 1 & Strong IZ (> $25 \mathrm{~mm})$ & Strong IZ (> $25 \mathrm{~mm})$ & Strong IZ (> $25 \mathrm{~mm})$ & Strong IZ (> $25 \mathrm{~mm})$ \\
\hline 2 & Strong IZ (> $25 \mathrm{~mm})$ & Strong IZ (> $25 \mathrm{~mm})$ & Strong IZ (> $25 \mathrm{~mm})$ & Strong IZ (> $25 \mathrm{~mm})$ \\
\hline $3 a$ & Moderate $\mathrm{IZ}=15 \mathrm{~mm}$ & Moderate $\mathrm{IZ}=15 \mathrm{~mm}$ & $I Z=0.0$ & $\mathrm{IZ}=0.0$ \\
\hline $3 b$ & Weak $I Z=9 \mathrm{~mm}$ & $\mathrm{IZ}=0.0$ & $I Z=0.0$ & $I Z=0.0$ \\
\hline $3 c$ & $I Z=0.0$ & Strong IZ (> $25 \mathrm{~mm})$ & $I Z=0.0$ & $\mathrm{IZ}=0.0$ \\
\hline $3 d$ & Strong IZ (> $25 \mathrm{~mm})$ & Strong IZ (> $25 \mathrm{~mm})$ & Strong IZ (> $25 \mathrm{~mm})$ & Strong IZ (> $25 \mathrm{~mm})$ \\
\hline 4 & Strong IZ (> $25 \mathrm{~mm})$ & Strong IZ (> 25 mm) & Strong IZ (> $25 \mathrm{~mm})$ & Strong IZ (> $25 \mathrm{~mm})$ \\
\hline
\end{tabular}




\begin{tabular}{|c|c|c|c|c|}
\hline 5 & Strong IZ (> $25 \mathrm{~mm})$ & Weak $I Z=5 \mathrm{~mm}$ & Strong IZ (> $25 \mathrm{~mm})$ & Strong IZ (> $25 \mathrm{~mm})$ \\
\hline 6 & Strong IZ (> $25 \mathrm{~mm})$ & Strong IZ (> $25 \mathrm{~mm})$ & Strong IZ (> $25 \mathrm{~mm})$ & Strong IZ (> $25 \mathrm{~mm})$ \\
\hline 7 & Strong IZ (> $25 \mathrm{~mm})$ & Strong IZ (> $25 \mathrm{~mm})$ & $I Z=0.0$ & $\mathrm{IZ}=0.0$ \\
\hline $1^{\prime}$ & Strong IZ (> $25 \mathrm{~mm})$ & Strong IZ (> $25 \mathrm{~mm})$ & Strong IZ (> $25 \mathrm{~mm})$ & Strong IZ (> $25 \mathrm{~mm})$ \\
\hline
\end{tabular}

Weakly active: less than $10 \mathrm{~mm}$, Moderately active: $10-20 \mathrm{~mm}$, Highly active: $20-25 \mathrm{~mm}$, Strong active: more than $25 \mathrm{~mm}$

\section{REFERENCES}

[1] Czerpack, R., Skolska, S., 1982. Med. Dosw. Microbiol. 34, 37-50, [Chem. Abstr. 1983, 98, 50232].

[2] Jund, L., Corse, J., King, A.S., Bayne, H., Mihrag, K., 1971. Phytochemistry, 10, 2971-2974.

[3] El-Ansary, S.L., Aly, E.I., Halem, M.A., 1992. J. Pharm. Sci. 33, 379-390.

[4] Reddy, Y.D., Somayoujulu, V.V., 1981. J. Ind. Chem. Soc. 58, 599-601.

[5] Rajyalakshmi, G., Rama, N.R.A., Sarangapani, M., 2011. Saudi Pharmaceutical Journal 19, 153-158.

[6] Wkrner, W., 1953. J. Org. Chem. 18, 1333-1337.

[7] Bhamaria, R.P., Bellare, R.A., Deliwala, C.V., 1968. Indian J. Exp. Biol. 6, 62-63.

[8] Abdel-Al, E.H., Al-Ashamawi, M.I., Abd El Fattah, B., 1983. Die Pharmazie 38, 833-838.

[9] Parmer, S.S., Kumar, R., 1963. J. Med. Chem. 11, 635-636.

[10] Gupta, A.K.S., Garg, M., Chandra, U. J. 1979. Indian Chem. Soc. 56, 1230-1232.

[11] Mansour, A.K., Eid, M.M., Khalil, N.S.A.M., 2003. Molecules, 8, 744-755.

[12] Milan, C., Maja, M., Bojan, S., Elizabeta, H.S., Valentina, R. 2010. Molecules 15, 6795-6809.

[13] Roshan, A., M. ZIA-UL-HAQ, Rukhsana, J., 1996. Tr. J. of Chemistry 20, 186-193.

[14] Mohamed, S.M., Nasser, M.A., Othman, Y.A.. 2013. Journal of Chemistry Article ID 183130, 7 pages.

[15] Alka, C., Sharma P.K., Niranjan, K., 2011. International Journal of Chem. Tech. Research 1, 11-17.

[16] Edina, M., Frigyes, W., György, K., Szabolcs L., Laszlo Ö., 2010. O.P.B. Sci. Bull. Series B. 72, Iss. 2.

[17] Govori, S., Rapic, V., Leci, O., Cacic, M., Tabakovic, I. J. 1996. Heterocyclic. Chem. 33, 351-354.

[18] Lacan, M., Cacic, M., Guslo, D., 1981. Acta Pharma Jugosl, 31, 47-51.

[19] Cacic, M., Trkovnik, M., Has-Schon, E. J., 2002. Heterocyclic. Chem. 40, 833-836.

[20] Gulay, S., Erhan, P., Melike, E., Meral, O.I., 2002. Farmaco. 57, 539-542.

[21] Harish, K., Sadique, A.J., Suroor, A.K., Mohammad, A., 2008. European Journal of Medicinal Chemistry 43, 26882698.

[22] Keshari, K.J., Adul, S., Yatendra, K., Mohd, S., Ratan, I.K., Jainendra, J., Vikash, K., Priyanka, S. 2010. European Journal of Medicinal Chemistry, 45, 4963-4967.

[23] Manjunatha, K., Boja, P., Prajwal, L.L., Jennifer, F., Suchetha Kumari, N., 2010. European Journal of Medicinal Chemistry, 45, 5225-5233.

[24] Pushpan, P., Boja, P., Chandrashekhar, C., Sunil, K.B., 2012. European Journal of Medicinal Chemistry 53, $203-210$.

[25] Vichai, V., Kirtikara, K., 2006. Nat. Protoc. 1, 1112-1116.

[26] Murray, PR., Baron, EJ., Pfaller, MA., Tenover, F.C., Yolken, H.R., 1995. Manual of clinical microbiology, 6th Ed. ASM Press, Washington DC 15-18.

[27] Olurinola, PF., 1996. A laboratory manual of pharmaceutical microbiology, Idu, Abuja, Nigeria, 69-105. 